\title{
Inverse effects of oxytocin on attributing mental activity to others in depressed and healthy subjects: a double-blind placebo controlled fMRI study
}

\section{David Pincus ${ }^{1}$, Samet Kose ${ }^{2,3,4}$, Ashley Arana ${ }^{2}$, Kevin Johnson ${ }^{2,3}$, Paul S. Morgan ${ }^{3,5}$, Jeffrey Borckardt ${ }^{2,3}$, Tal Herbsman ${ }^{6}$, Fran Hardaway ${ }^{2}$, Mark S. George ${ }^{2,3,5,78}$, Jaak Panksepp ${ }^{9}$ and Ziad Nahas ${ }^{2,3,7 *}$}

' SUMMA Health, Akron, OH, USA

2 Mood Disorders Program and Brain Stimulation Laboratory, Department of Psychiatry, Medical University of South Carolina, Charleston, SC, USA

${ }^{3}$ Center for Advanced Imaging Research, Medical University of South Carolina, Charleston, SC, USA

${ }^{4}$ Department of Psychiatry, Vanderbilt University, Nashville, TN, USA

${ }_{5}^{5}$ Department of Radiology, Medical University of South Carolina, Charleston, SC, USA

${ }^{6}$ Department of Psychiatry, The Dean Clinics, Madison, WI , USA

7 Department of Neurosciences, Medical University of South Carolina, Charleston, SC, USA

${ }^{8}$ Ralph H. Johnson VA, Charleston, SC, USA

${ }^{9}$ Washington State University, Pullman, WA, USA

\section{Edited by:}

Zafiris J. Daskalakis, University of Toronto, Canada

\section{Reviewed by:}

Paul Croarkin, UT Southwestern

Medical Center, USA

Tarek Rajji, Centre for Addiction and

Mental Health, Canada

*Correspondence:

Ziad Nahas, Department of Psychiatry and Behavioral Sciences, Institute of

Psychiatry, 67 President St.,

Charleston, SC 29425, USA

e-mail: nahasz@musc.edu
Background: Oxytocin is a stress-attenuating and pro-social neuropeptide. To date, no study has looked at the effects of oxytocin in modulating brain activity in depressed individuals nor attempted to correlate this activity with attribution of mental activity in others. Method: We enrolled 10 unmedicated depressed adults and 10 matched healthy controls in a crossover, double blind placebo controlled fMRI 40 i.u. intra-nasal oxytocin study (20 i.u. per nostril). Each subject performed reading the mind in the eyes task (RMET) before and after inhalation of oxytocin or placebo control for a total of 80 scans. Results: Before oxytocin administration, RMET engaged the medial and lateral prefrontal cortex, amygdala, insula and associative areas. Depressed subjects showed increased anterior ventral activation for the RMET minus gender identification contrast whereas matched controls showed increased dorsal and frontal activity. Compared to placebo, oxytocin in depressed subjects showed increased activity in the superior middle frontal gyrus and insula, while controls exhibited more activity in ventral regions. Oxytocin also led to inverse effects in reaction times on attribution task between groups, with controls getting faster and depressed individuals slower to respond. Conclusion: Depression is associated with increased paralimbic activity during emotional mental attribution of others, appearing to be distinctly modulated by oxytocin when compared to healthy controls. Further studies are needed to explore long-term exposure to pro-social neuropeptides on mood in depressed populations and assess their clinical relevance.

Keywords: oxytocin, fMRI, mentalization, theory of mind, depression

\section{INTRODUCTION}

Mentalization, a developmentally derived ability to affectively and cognitively infer the mental state of others is crucial to sociality and is enhanced by propitious genetics and secure attachments (ChoiKain and Gunderson, 2008; Hartmann, 2009). Mentalization allows individuals to estimate others' states of mind, and one's reactions and reflections upon that approximation represent an advantageous ability given that humans need good social bonds for maximum health, adaptation, and richer relationships. It begins in infancy and is facilitated by positive social interactions (Fonagy and Target, 2007) and early attachment experiences (Meins et al., 2002).

Although little is known about the neurophysiology of such experiences, there is evidence that oxytocin, a neuropeptide, promotes positive social interactions and preferences (Hurlemann et al., 2010, Winslow and Insel, 2004; Zak et al., 2005), enhances socially reinforced learning and faces recognition (Marsh et al., 2010, Guastella et al., 2008), exerts anxiolytic effects and is a key modulator of early mother-infant bonding and maternal caretaking (Panksepp, 2005).
It is increasingly released in affectively rich dyadic settings such a when social attunement occurs between mother and infant, in breastfeeding, early skin to skin contact, and later in sexual intercourse and climax (Nelson and Panksepp, 1998; Uvnas-Moberg, 1998). Conversely, low levels of oxytocin have been linked to depression (Bell, 2006), with patients experiencing and observed to possess "dis-affiliation" with others. Also, critical social events marked with insecure attachment or losses of significant others often precipitate depressive symptoms (Anisman, 1984). Functional neuroimaging studies consistently find abnormal ventral limbic and paralimbic activity in depression (Price and Drevets, 2009) - the very same regions implicated in the mediation of social relatedness and mentalization (Choi-Kain and Gunderson, 2008; Hartmann, 2009).

Theory of mind ( $\mathrm{ToM})$ is a research and conceptual rubric of an "imagined involvement" that was derived from studies of autism. Mentalization is a higher order capacity, either deficient in clinical populations or progressively enhanced with better caretaking and affective attunement. The failure to evolve mentalization partially 
explains pervasive developmental disorders (Ozonoff et al., 1991; Happe, 1994; Happe et al., 1996), schizophrenia (Pilowsky et al., 2000) and reduced social interactions in depression (Uekermann et al., 2008). Lower social function is associated with higher risks for symptom recurrence 1 year after recovering from a major depressive episode (Uekermann et al., 2008). The interest in mirror neurons (Rizzolatti and Fabbri-Destro, 2008) provides a potential neural basis for intent prediction of others. Imaging studies using single photon emission computed tomography (SPECT) (Vogeley et al., 2001b), positron emission tomography (PET) (Happe et al., 1996) or fMRI (Gallagher et al., 2000) have utilized different ToM paradigms (Stuss et al., 2001; Vogeley et al., 2001a) with mixed results. These studies do, however, shed light upon functional networks correlated with mentalization and the social nature of the self and point to the amygdala, the superior temporal gyrus, and medial and orbito-frontal cortex as keys to this high-order mental process.

A "Reading the Mind in the Eyes test" (RMET) (Baron-Cohen et al., 2001) was developed to study emotional mental attribution. This task requires the subject to infer the mental state of an individual from the expression in their eyes and immediately surrounding areas, a measure of imagined involvement as opposed to an appraisal task. Brain regions previously shown to be significantly activated by the task include fronto-temporal neocortical regions, such as left dorsolateral prefrontal cortex, medial frontal cortex and superior temporal gyrus as well as non-cortical regions such as left amygdala, left hippocampus, bilateral insula and left striatum, with some of the brain changes being culture specific (Johnston et al., 2005; Baron-Cohen et al., 2006; Williams et al., 2008).

To date, no studies have investigated the functional neuroanatomy of mentalization in depression nor the effects of oxytocin in modulating brain activity in depressed individuals. Accordingly, we used the RMET task and an intra-nasal oxytocin challenge to investigate mentalization in unmedicated depressed patients and healthy controls. We hypothesized that depressed patients may show greater limbic activity during the mentalization task compared to matched controls, and that oxytocin will lead to a significant decrease in ventro-limbic activity when compared to placebo.

\section{MATERIALS AND METHODS \\ DESIGN}

This is a randomized double-blind crossover design to investigate the acute effects of intra-nasal oxytocin on mentalization in unmedicated depressed patients and matched controls. Primary outcome measures consisted of functional blood oxygen level dependent MRI (BOLD fMRI) scans. Secondary outcome measures were behavioral measures (speed of reaction time). This study was approved by the Medical University of South Carolina (MUSC) Institutional Review Board funded by a grant from the Hope for Depression Research Foundation, and collaboratively conducted with the MindBrain Consortium at Summa Hospitals, Akron, Ohio.

\section{SUBJECTS}

Subjects were recruited from the MUSC outpatient psychiatric services, the Mood Disorders Program (MDP) and through local advertisement. We screened 15 Healthy and 20 Depressed subjects and enrolled 22 adult subjects. All subjects signed written informed consent. Depressed patients met criteria for major depressive episode by Structured Clinical Interview for DSM-IV Axis I Disorders (SCID-I) criteria of less than 24 months in duration with an antidepressant treatment history form (ATHF) severity one or less failed trials. Healthy controls were screened for any active DSM-IV-TR Axis I diagnosis (with the exception of caffeine or nicotine abuse). Subjects were off all psychotropic medications for at least 2 weeks prior to first scanning session. Subjects were excluded if they were medically unstable or had an active neurodegenerative or epilepsy disorder. All presented data are from subjects who completed a baseline clinical assessment visit along with two scanning sessions over a maximum of 3 weeks period.

\section{SCANNING AND DRUG INHALATION PROCEDURES}

At each scanning session, subjects underwent two identical blood oxygentation dependant (BOLD) fMRI scans before and approximately 10 min after inhalation of study drug (20 IU oxytocin or placebo per nostril; Lee-Silsby Pharmacy, Cleveland, OH, USA). The oxytocin dose is comparable to that used previously in human studies of behavior (Epperson et al., 1996a,b; Ditzen et al., 2007). Randomization and subject's assignment were performed by MUSC Investigational Drug Services and the McClellan Banks Pharmacy. Data was unmasked after study completion and all data were locked. Subjects abstained from alcohol and nicotine for $12 \mathrm{~h}$ before the experiment.

\section{READING THE MIND IN THE EYES PARADIGM}

We adapted the reading the mind in the eyes paradigm (RMET) for presentation in the scanner and an event-fMRI design. We used the Integrated Functional Imaging System (IFIS-SA) (Invivo, Orlando, FL, USA) to visually present the paradigm and record responses of the participants. The RMET task consisted of three conditions: affect attribution of faces cropped around the eyes in rectangular shape (referred in the text as RMET), gender identification of the same photographs, and a basic motor cued response. Possible attribute adjectives, gender attributes ("male," "female") and motor cues ("yes," "no") were positioned at the four corners with optimal or normed responses equally randomized for all presentations. Participants pressed a hand-pad button to indicate their attribute of choice. On the hand-pad, the left and right index fingers corresponded to the top left and top right corners of images, and the left and right thumbs corresponded to the bottom left and bottom right corners of images. This entire paradigm consisted of 32 events for each condition ( 96 events total presented in a fixed-randomized order) and lasted approximately $769 \mathrm{~s}$. The duration of each event lasted $7 \mathrm{~s}$, separated by a jittered inter-stimulus black screen [interstimulus interval (ISI) range 6-4753 ms, ISI average $=1008 \mathrm{~ms}$ ].

\section{IMAGE ACQUISITION}

Images were acquired using a 3T MRI scanner (Intera, Philips Medical System, Netherlands) with a SENSE parallel imaging head-coil. A sagittal reference image was first acquired, to guide positioning of the functional scans along the anterior commissure-posterior commissure line. For scanning during the RMET paradigm, echoplanar (EPI) transverse images were acquired with the following parameters: 414 volumes, repetition time $=1867 \mathrm{~ms}$, echo time $=30 \mathrm{~ms}$, flip angle $=90^{\circ}$, field of view $=208 \mathrm{~mm}$, matrix $=64 \times 64$, SENSE factor $=2,32$ slices, $3.25 \mathrm{~mm}$ with no gap, yielding a voxel size of $3.3 \times 3.3 \times 3.25 \mathrm{~mm}^{3}$. 


\section{DATA ANALYSIS}

\section{Demographics and psychological measures}

Descriptive statistics were performed using SPSS 16.0 for Macintosh (SPSS Inc., Chicago, IL, USA) and included mean, standard deviation, Student's $t$-test, Pearson Chi-square test. All tests were twotailed at 0.05 significant level and assumed equal variance.

\section{fMRI data processing methods}

fMRI image data was first transferred to a workstation where it was converted into Analyze format using MRICro ${ }^{1}$ and then subjected to the following pre-statistics processing steps using FMRI FEAT (FMRI Expert Analysis Tool) Version 5.92, part of FSL (FMRIB's Software Library ${ }^{2}$. Each dataset was spatially normalized into an high resolution structural scan MNI template with FLIRT (Jenkinson and Smith, 2001; Jenkinson et al., 2002) We applied MCFLIRT for motion correction (Jenkinson et al., 2002) and reviewed all datasets for remaining motion, which was less than $0.5 \mathrm{~mm}$ along all three major axes $(x, y, z)$ for all subjects. In addition, we applied non-brain removal using BET (Smith, 2002); spatial smoothing using a Gaussian kernel of FWHM 8.0 mm; grand-mean intensity normalization of the entire $4 \mathrm{D}$ dataset by a single multiplicative factor and highpass temporal filtering (Gaussian-weighted leastsquares straight line fitting, with sigma $=50.0 \mathrm{~s}$ ).

\section{Behavioral data}

Individual responses collected by IFIS during RMET were collated, checked for missing responses and collated in an Excel spreadsheet. Reaction Time (RT) and Accuracy of Response (AC) were noted. Final analysis included mean, standard deviation, Student's $t$-test, and Pearson Chi-square test. All tests were two-tailed and assumed equal variance.

For mixed models examining the effects of drug by time, pre-drug administration conditions were coded in the dataset as " 0 ," post-drug were coded as " 1 ," placebo conditions were coded as " 0 " and oxytocin conditions were coded as "1." Hierarchical Linear Modeling (HLM) was implemented using PROC MIXED in SAS (SAS Institute Inc., Cary, NC) to assess the linear effect of the interaction of "time" (pre to post-drug administration) and "treatment type" (oxytocin versus placebo) on reaction time and accuracy of response between groups (thereby by testing a series of $2 \times 2 \times 2$ mixed models). HLM has been shown to handle nested models with serially dependent data points and randomly distributed missing values appropriately (Raudenbush and Chan, 1993; de Leeuw and Kreft, 1995; Hoffman and Gavin, 1998; Nezlek and Zyzniewski, 1998). Additionally, this approach is growing in popularity among clinical and educational researchers due to its flexibility and appropriateness for data typically encountered in clinical and social science research (Sliwinski and Hall, 1998; Kuo et al., 2000; McGuigan et al., 2003). The covariance structure was "unstructured" and the estimation method was REML.

\section{fMRI statistical analysis}

Time-series statistical analysis was carried out using FILM with local autocorrelation correction (Woolrich et al., 2001). Potentially confounding motion over time was regressed out. $Z$ (Gaussianized T/F)

${ }^{1}$ http://www.sph.sc.edu/comd/rorden/mricro.html ${ }^{2}$ www.fmrib.ox.ac.uk/fs statistic images were thresholded using clusters determined by $Z>2.3$ and a (corrected) cluster significance threshold of $p=0.05$ (Worsley, 2001) for identification of voxels with statistically significant activation during three conditions in individual subjects (fixed effect). We then performed a group mean (baseline depressed + matched controls) and unpaired $t$-tests between oxytocin and placebo conditions (random effect; FLAME $1+2$ option).

\section{RESULTS}

Eight unmedicated depressed subjects (eight females, four AfricanAmericans, four Caucasians) and nine matched controls (eight females, five African-Americans, four Caucasians) completed all assessments required for this study. The mean age for depressed subjects was 35.5 years $(S D=10.62)$, ranging from 26 to 60 , and for controls, 36.4 years $(\mathrm{SD}=11.40)$, ranging from 25 to 59 . Mean years of education post high school were $2.56(\mathrm{SD}=1.95)$ for depressed subjects and 4.22 (SD = 1.79) for controls. Co-morbidities of depressed subjects included a past history of alcohol dependence $(n=2)$, social phobia $(n=1)$, obsessive compulsive disorder $(n=1)$, panic disorder without agoraphobia $(n=2)$, anxiety $(n=1)$, and anorexia nervosa $(n=1)$. All subjects were right handed. Sixteen subjects were employed either part or full time, and one was a homemaker. The patient cohort had significant depressive and anxiety symptoms compared to healthy controls and tended to show reduced capacity for empathy but not? overall emotional intelligence as measured by the Mayer-Salovey-Caruso Emotional Intelligence Test (MSCEIT) (Mayer et al., 2003) (See Table 1). There was a significant inverse correlation between subjective depressive symptoms (IDS-SR) and empathy quotient $\left(\mathrm{df}=21, r^{2}=0.35, p=0.004\right)$.

\section{BEHAVIORAL RESULTS \\ Reaction time}

Figure 1 shows reaction time slope changes with intra-nasal oxytocin in healthy controls and depressed subjects across RMET, gender attribution, and motor cued response. A significant effect was found for the slope of the drug $\times$ time $\times$ group reaction time $(\mathrm{RT})$ interaction during $\operatorname{RMET}[F(1,2551)=5.99, p=0.015]$. A decreased RT slope was found in healthy matched controls $[t(2551)=2.20, p=0.028]$ relative to an increased slope in depressed subjects $[t(2551)=2.45$, $p=0.015]$, suggesting that oxytocin administration was associated with faster reaction times in the healthy cohort and slower reaction times in the depressed cohort after controlling for placebo and predrug performance. A significant effect was also found for the slope of the drug $\times$ time $\times$ group RT interaction during gender attribution $[F(1,2510)=14.82, p<0.0001]$. Again, a decreased RT slope was found in healthy matched controls $[t(2510)=4.27, p<0.0001]$ relative to an increased slope in depressed subjects $[t(2510)=3.85$, $p<0.0001]$. Again, this suggests that oxytocin administration was associated with faster reaction times in the healthy cohort and slower reaction times in the depressed cohort after controlling for placebo and pre-drug performance. Lastly, a significant effect was found for the slope of the drug $\times$ time $\times$ group $\mathrm{RT}$ interaction during motor cued response $[F(1,2507)=6.87, p=0.009]$. Oxytocin administration was associated with decreased RT slope in healthy matched controls $[t(2507)=2.92, p=0.004]$ relative to an increased slope in depressed subjects $[t(2507)=2.62, p=0.009]$ after controlling for placebo and pre-treatment performance. The mean effect size 
Table 1 | Demographics and clinical characteristics of unmedicated depressed subjects and their matched healthy controls. Significant differences were found in depressive symptoms (HDRS, IDS-SR, ANPS-Sadness), anxiety symptoms (STAY-I and ANPS-Fear), and capacity to care (ANPS-Care). Note that a trend for differences on Empathy Quotient and ANPS-Spirituality.

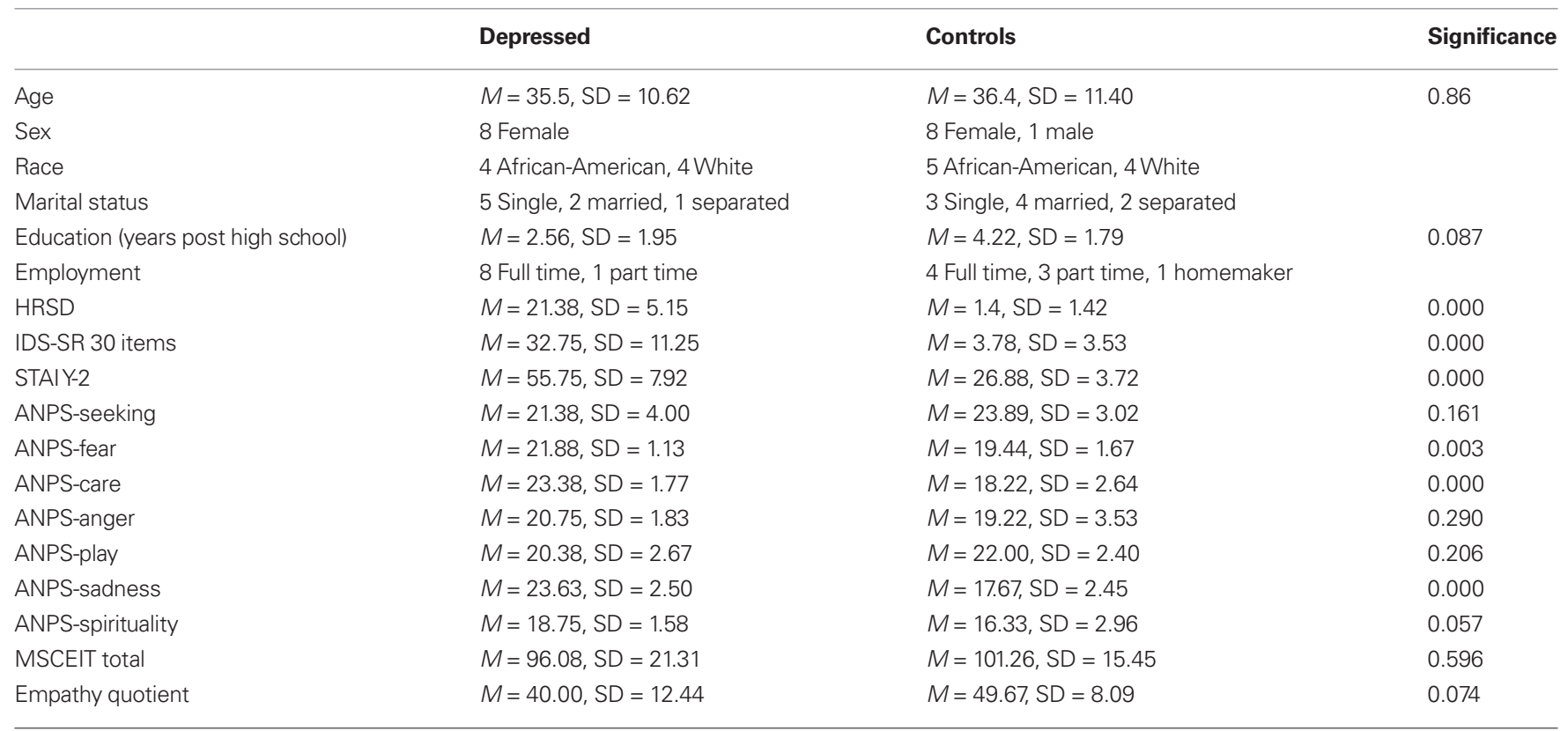

Table 2 | Reaction times (RT) in milliseconds and accuracy of response (AC) in unmedicated depressed subjects and their matched healthy controls during fMRI sessions. Data represent mean and standard deviation across three variables (RMET, gender attribution, and cued-motor response) and four conditions (pre-oxytocin, post-oxytocin, pre-placebo, and post-placebo).

\begin{tabular}{|c|c|c|c|c|}
\hline & Pre-placebo & Pre-oxytocin & Post-placebo & Post-oxytocin \\
\hline \multicolumn{5}{|l|}{ RT RMET } \\
\hline Matched controls & $4396.3 \pm 1499.8$ & $4198.5 \pm 1728.6$ & $4092.5 \pm 1560.8$ & $4222.8 \pm 1293$ \\
\hline \multicolumn{5}{|l|}{ RT gender attribution } \\
\hline Matched controls & $2168.7 \pm 958.3$ & $2126.1 \pm 853.3$ & $2023.2 \pm 950.7$ & $1936.4 \pm 710.3$ \\
\hline Matched controls & $1416.8 \pm 592$ & $1464.4 \pm 730.2$ & $1363.1 \pm 791.9$ & $1351.7 \pm 465.6$ \\
\hline Depressed & $1214.7 \pm 472.9$ & $1257.6 \pm 505$ & $1134.6 \pm 461.8$ & $1240.5 \pm 395.9$ \\
\hline \multicolumn{5}{|l|}{ AC RMET } \\
\hline Matched controls & $60.6 \%$ & $59.4 \%$ & $64.2 \%$ & $67.9 \%$ \\
\hline Depressed & $65.8 \%$ & $67 \%$ & $67 \%$ & $70.6 \%$ \\
\hline Matched controls & $98.1 \%$ & $94.1 \%$ & $93.1 \%$ & $97.8 \%$ \\
\hline Depressed & $96.9 \%$ & $97.5 \%$ & $95.6 \%$ & $97.2 \%$ \\
\hline
\end{tabular}

(Cohen's d) across all RT for oxytocin is small at 0.18 . Table 2 shows the mean values (and standard error) for each variable pre to postoxytocin or placebo.

\section{Accuracy of response}

There were no significant changes with accuracy of response (AR) across variables or conditions. Table 2 summarizes the findings described above and Table 2 shows the mean values (and stand- ard error) for each variable pre to post-oxytocin or placebo. No significant antidepressant effects of oxytocin were observed in this provocation-paradigm study.

\section{FUNCTIONAL IMAGING}

The corresponding brain atlas coordinates and the fMRI activations for healthy controls and depressed subjects are presented in Table 3. 
Table 3 | fMRI activations Talairach coordinates and corresponding z-scores.

\begin{tabular}{|c|c|c|c|c|c|c|}
\hline z-Score & $x$ & $y$ & $z$ & Left/right & Region & Brodmann area \\
\hline \multicolumn{7}{|c|}{ Healthy controls $n=9$} \\
\hline 5.7 & -46 & 14 & 26 & Left & Middle frontal gyrus & Brodmann area 9 \\
\hline 5.57 & 22 & -64 & 54 & Right & Superior parietal lobule & Brodmann area 7 \\
\hline 5.51 & -6 & 6 & 60 & Left & Superior frontal gyrus & Brodmann area 6 \\
\hline 5.19 & -20 & -64 & 58 & Left & Superior parietal lobule & Brodmann area 7 \\
\hline 4.87 & -32 & -84 & -10 & Left & Inferior occipital gyrus & Brodmann area 18 \\
\hline \multicolumn{7}{|c|}{ RMET minus gender group mean } \\
\hline 4.5 & -10 & 26 & 56 & Left & Superior frontal gyrus & Brodmann area 6 \\
\hline 4.39 & -10 & 12 & 6 & Left & Caudate & Caudate Body \\
\hline 4.32 & -6 & 54 & 40 & Left & Medial frontal gyrus & Brodmann area 9 \\
\hline 5.79 & -46 & 26 & -10 & Left & Inferior frontal gyrus & Brodmann area 47 \\
\hline 5.21 & -54 & 22 & 8 & Left & Inferior frontal gyrus & Brodmann area 45 \\
\hline 4.52 & -52 & -48 & 2 & Left & Middle temporal gyrus & Brodmann area 22 \\
\hline 3.79 & -42 & 10 & -36 & Left & Middle temporal gyrus & Brodmann area 21 \\
\hline
\end{tabular}

Unmedicated depressed $n=8$

RMET group mean

$\begin{array}{lllll}5.29 & 10 & 18 & 40 & \text { Right } \\ 4.83 & 36 & 22 & 0 & \text { Right } \\ 4.66 & -22 & -58 & 44 & \text { Left } \\ 4.55 & 30 & -4 & 50 & \text { Right } \\ 4.35 & -10 & 8 & 2 & \text { Left } \\ \text { RMET minus gender } & -58 \text { roup mean } & & & \\ 4.3 & -58 & 22 & 4 & \text { Left } \\ 4.14 & -48 & 14 & -161 & \text { Left } \\ 4.11 & -40 & 28 & -10 & \text { Left } \\ 4.04 & -10 & 14 & -6 & \text { Left } \\ 2.3 & -14 & 10 & -22 & \text { Left }\end{array}$

DIRECT COMPARISONS BETWEEN INTRA-NASAL OXYTOCIN AND PLACEBO

Healthy matched controls $\boldsymbol{n}=\mathbf{9}$

RMET healthy oxytocin > placebo t-test

$\begin{array}{lll}3.93 & -6 & 6 \\ 3.82 & 12 & -4 \\ 2.66 & 14 & -2 \\ 3.73 & 28 & 32 \\ 3.72 & -8 & 0 \\ 3.67 & -10 & 32 \\ 3.67 & 30 & -24 \\ 4.24 & -40 & -30 \\ 3.94 & -50 & 8 \\ 3.7 & -56 & -8 \\ 2.7 & -4 & 18 \\ 2.69 & -4 & 48\end{array}$

Gender healthy oxytocin $>$ placebo t-test

Motor healthy oxytocin > placebo t-test

$\begin{array}{lll}3.99 & 50 & -27 \\ 3.76 & 16 & -30\end{array}$

$\begin{array}{ll}-8 & \text { Left } \\ -20 & \text { Right } \\ -22 & \text { Right } \\ -18 & \text { Right } \\ -6 & \text { Left } \\ -6 & \text { Left } \\ -24 & \text { Right } \\ -10 & \text { Left } \\ -18 & \text { Left } \\ -6 & \text { Left } \\ -12 & \text { Left } \\ -14 & \text { Left }\end{array}$

No significant differences

$10 \quad$ Right
Caudate

Parahippocampal gyrus

Amygdala

Inferior frontal gyrus

Lentiform nucleus

Anterior cingulate

Parahippocampal gyrus

Caudate

Superior temporal gyrus

Superior temporal gyrus

Anterior cingulate gyrus

Anterior cingulate gyrus
Brodmann area 32 Brodmann area 13 Brodmann area 7 Brodmann area 6 Caudate Head

Brodmann area 45 Brodmann area 38 Brodmann area 47 Caudate head Brodmann area 25
Caudate head

Brodmann area 34

Brodmann area 47

Brodmann area 24 Brodmann area 35 Caudate tail Brodmann area 38 Brodmann area 22 Brodmann area 25 Brodmann area 32 
Table 3 | (Continued)

\begin{tabular}{|c|c|c|c|c|c|c|}
\hline z-Score & $x$ & $y$ & $z$ & Left/right & Region & Brodmann area \\
\hline 3.72 & 60 & -21 & 9 & Right & Superior temporal gyrus & Brodmann area 42 \\
\hline 3.65 & 47 & -31 & 15 & Right & Superior temporal gyrus & Brodmann area 41 \\
\hline 3.62 & 51 & -30 & 15 & Right & Superior temporal gyrus & Brodmann area 41 \\
\hline \multicolumn{7}{|c|}{ Depressed unmedicated $n=8$} \\
\hline 3.79 & 54 & 8 & 2 & Right & Insula & Brodmann area 13 \\
\hline 3.77 & 56 & 12 & 6 & Right & Precentral gyrus & Brodmann area 44 \\
\hline 3.73 & 50 & 18 & -14 & Right & Inferior frontal gyrus & Brodmann area 47 \\
\hline 3.71 & 58 & 8 & -10 & Right & Superior temporal gyrus & Brodmann area 22 \\
\hline 3.76 & -4 & 36 & 16 & Left & Anterior cingulate & Brodmann area 24 \\
\hline 3.73 & -64 & -52 & 24 & Left & Superior temporal gyrus & Brodmann area 22 \\
\hline 3.69 & -58 & -48 & 32 & Left & Supramarginal gyrus & Brodmann area 40 \\
\hline 3.65 & -52 & -32 & 8 & Left & Superior temporal gyrus & Brodmann area 22 \\
\hline 3.54 & 30 & 58 & -6 & Right & Superior frontal gyrus & Brodmann area 10 \\
\hline 3.52 & 44 & 36 & 28 & Right & Middle frontal gyrus & Brodmann area 9 \\
\hline 3.41 & 28 & 44 & 34 & Right & Middle frontal gyrus & Brodmann area 8 \\
\hline 3.35 & 34 & 52 & 12 & Right & Middle frontal gyrus & Brodmann area 10 \\
\hline 3.33 & 24 & 48 & 22 & Right & Superior frontal gyrus & Brodmann area 9 \\
\hline
\end{tabular}

RMET minus gender depressed oxytocin > placebo t-test

Gender depressed oxytocin > placebo t-test

Motor depressed oxytocin > placebo t-test

$\begin{array}{lll}3.51 & 0 & 58 \\ 3.41 & -18 & 56 \\ 3.39 & 4 & 60 \\ 3.44 & -2 & 58 \\ 3.47 & 2 & 56 \\ 3.59 & -2 & 34 \\ 3.6 & 8 & 6\end{array}$

No significant differences

No significant differences

$\begin{array}{ll}\text { Left } & \text { Medial frontal gyrus } \\ \text { Left } & \text { Superior frontal gyrus } \\ \text { Right } & \text { Medial frontal gyrus } \\ \text { Left } & \text { Medial frontal gyrus } \\ \text { Left } & \text { Medial frontal gyrus } \\ \text { Left } & \text { Superior frontal gyrus } \\ \text { Right } & \text { Medial frontal gyrus }\end{array}$

Brodmann area 9

Brodmann area 9

Brodmann area 8

Brodmann area 8

Brodmann area 6

Brodmann area 6

Brodmann area 6

\section{Baseline mean groups}

During the RMET task, at baseline and prior to inhaling study drug, depressed and normal control subjects showed statistically significant activations of the visual cortex, associated visual areas, the fusiform gyrus, and various limbic areas, along with the anterior cingulate. The depressed group showed significant activation in the right anterior cingulate gyrus (BA32), insula and middle frontal gyrus (BA6), left superior parietal lobe (BA7) and head of the caudate. The two groups were not significantly different in intensities or areas of activation on the mentalization task. They did however differ when contrasting RMET minus gender identification (see Figure 2). The depressed group showed predominantly ventral activations with left inferior frontal gyrus, superior temporal gyrus, subgenual cingulum and head of the caudate. The healthy controls showed activations in the superior and middle frontal gyri, the orbito-frontal cortex and middle temporal gyrus (BA21, 22).

\section{Oxytocin versus placebo}

Depressed group. During RMET, there was significant activation with oxytocin compared to placebo in bilateral superior temporal gyrus (BA38, 41, and 22), right insula, right precentral gyrus (BA44), bilateral cingulate gyri (BA32), left anterior cingulum (BA24), left supramarginal gyrus (BA40), right inferior frontal gyrus (BA47), right superior and middle frontal gyri (BA8, 9, and 10) (see Figure 3). On motor cues, oxytocin led to significant increases in bilateral superior and medial frontal gyri (BA6, 8 and 9). Gender identifications and RMET minus gender identifications did not differ between oxytocin and placebo conditions.

Matched healthy controls. During RMET, there was significant activation with oxytocin compared to placebo in right parahippocampal gyrus extending to the amygdala, inferior frontal gyrus (BA47), left head and tail of the caudate and superior temporal gyrus (BA22 


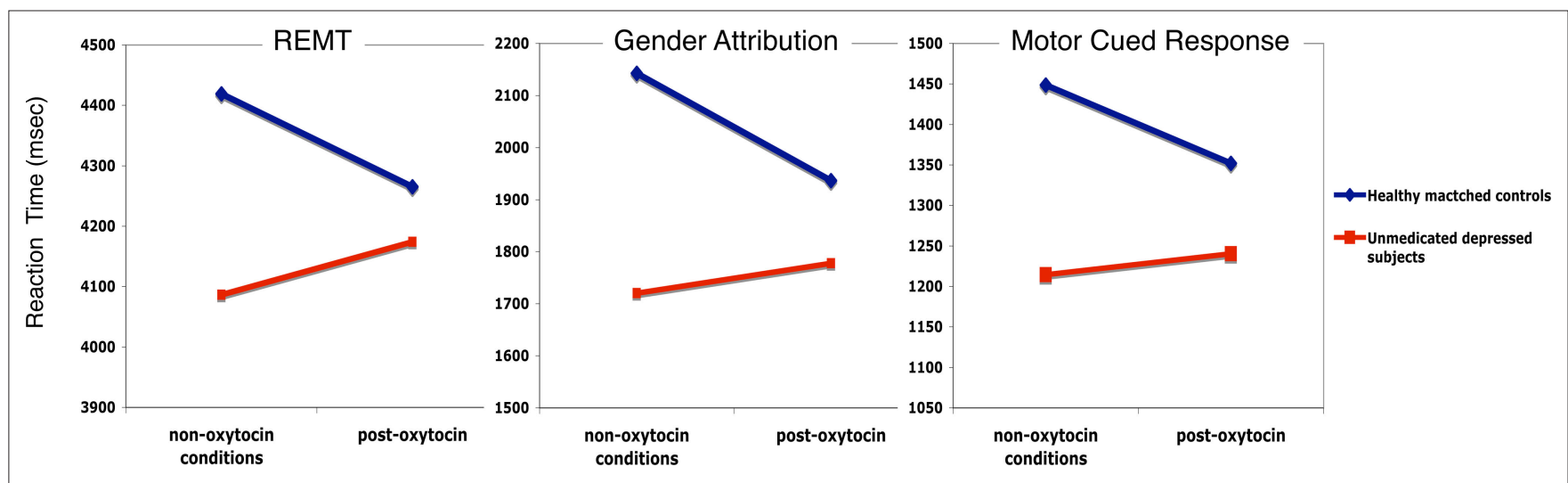

FIGURE 1 | Reaction time slope changes with intra-nasal oxytocin in healthy matched controls (blue) and depressed subjects (red) across RMET, gender attribution and motor cued response.

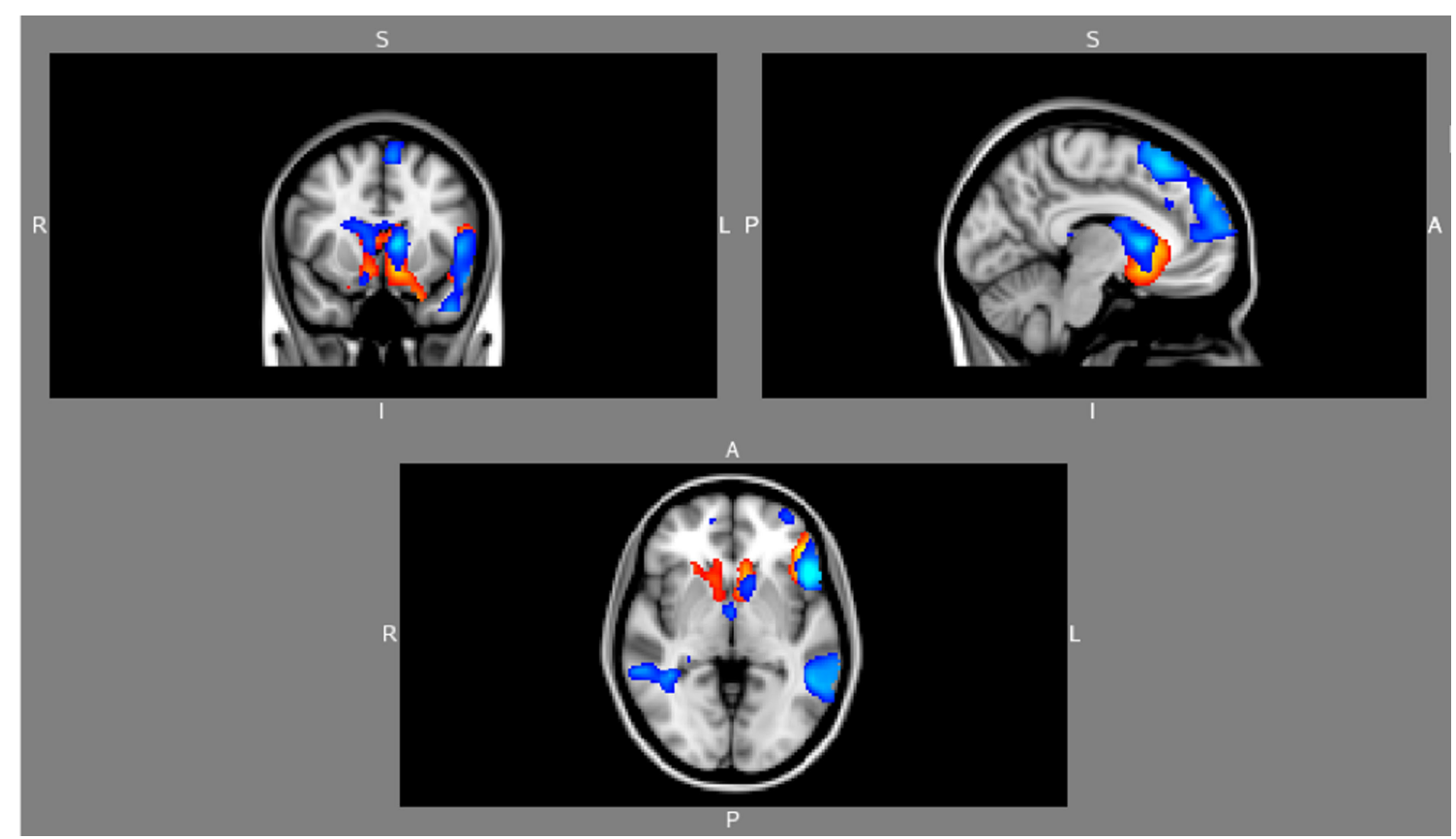

FIGURE 2 | Baseline RMET minus Gender Attribution: Predominant ventral activations in unmedicated depressed subjects at baseline (red) compared to superior frontal and temporal activations in healthy matched controls (blue).

and 38) (see Figure 4). On Motor Cues, oxytocin led to significant increases in right superior temporal gyrus (BA41 and 42) and right cerebellum. Gender identifications and RMET minus gender identifications did not differ between oxytocin and placebo conditions.

\section{DISCUSSION}

To our knowledge, this is the first study investigating intra-nasal oxytocin in unmedicated depressed and matched controls using fMRI. We employed RMET, a visual perceptual task that involves more affectively valenced decision making and mental attributions of other people. The task emphasizes affective "involvement" rather than cognitive "appraisal." At baseline, the two groups were significantly different in their distress symptoms and capacity to empa- thize. Along with activating attentional and visual networks, RMET also involved anterior ventromedial regions. Oxytocin differentially affected normal and depressed cohorts. In controls, oxytocin enhanced activation of ventromedial, amygdala, parahippocampal and semantic associative areas. Depressed subjects showed increases in higher order cognitive areas and insula. Oxytocin also showed opposite effects in reaction times with increased speed of response in healthy controls and a slowing among the depressed.

\section{MENTALIZING IN DEPRESSION}

The mentalization task is a novel paradigm in this population. It is relatively complex and recruits several complementary limbic, attention and high-level executive networks. Pathological states 


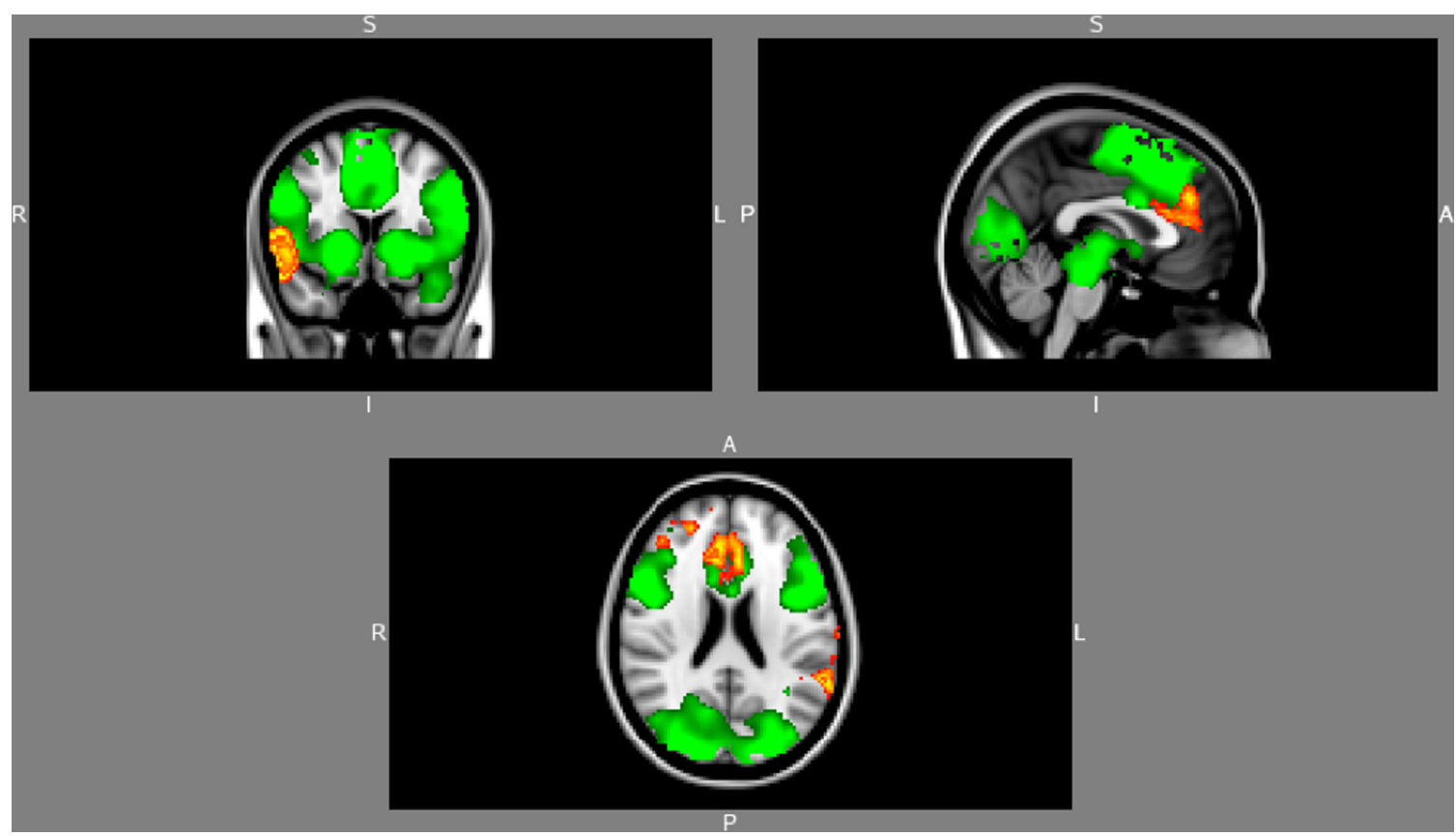

FIGURE 3 | Baseline (green) and added activations with oxytocin compared to placebo (red) in unmedicated depressed subjects during RMET.

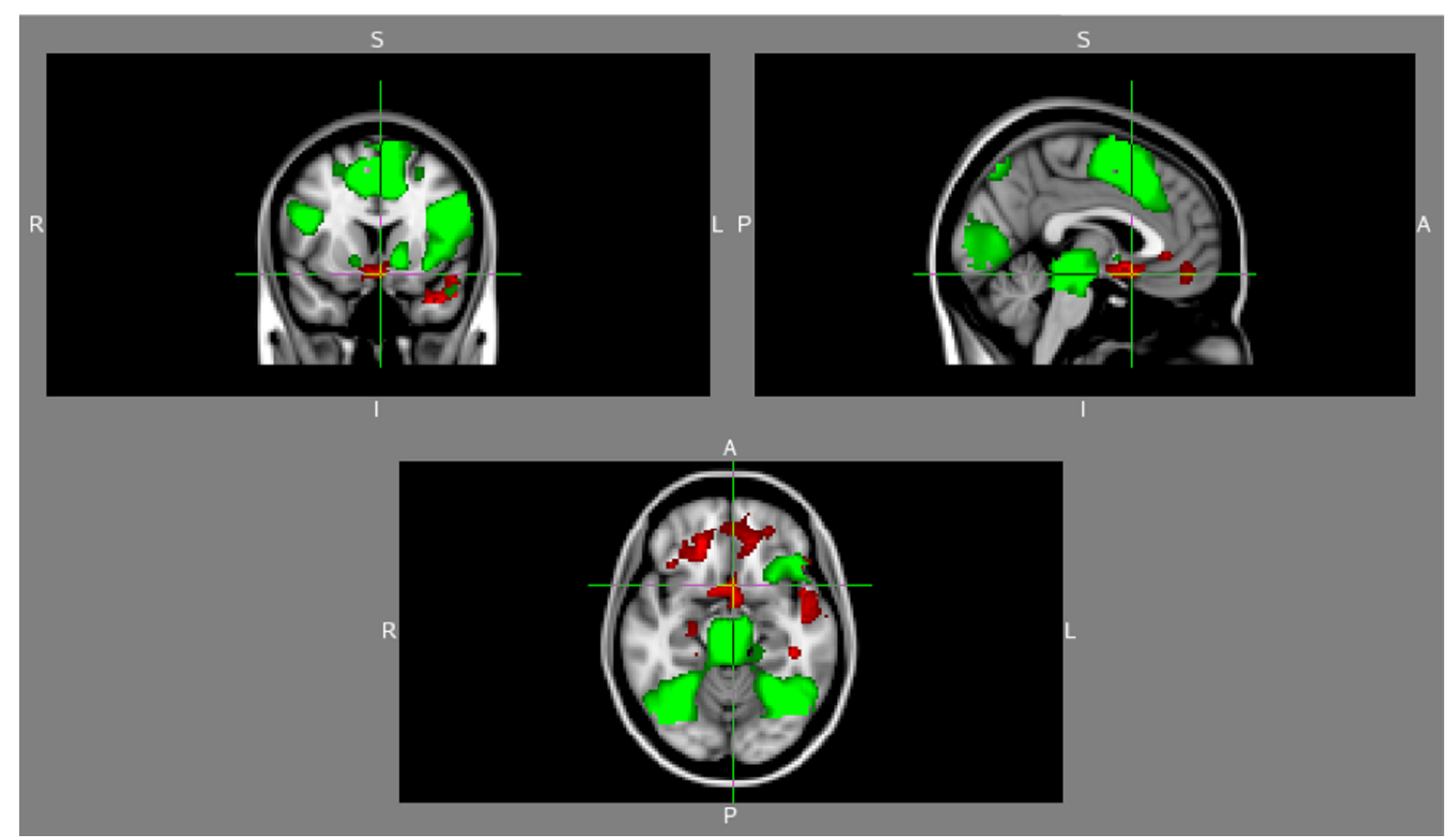

FIGURE 4 | Baseline (green) and added activations with oxytocin compared to placebo (red) in healthy matched controls during RMET.

such as depression degrade mentalization (Uekermann et al., 2008), perhaps by limiting emotional involvement with all things and people in their experiential world and reflection upon their internal world, functioning more in a purely cognitively appraised, distant or disconnected fashion. The results reported here suggest that depression may represent a homeostatic alteration in contributions of these systems to executing this task compared to healthy controls.
Many of the findings we report are in line with abundant existing evidence of limbic and executive dysfunction in depression (Green et al., 2007; Northoff, 2007). The distinct increase in ventro-limbic activity is also reminiscent of mood induction studies (George et al., 1995; Mayberg et al., 1999). But the direct consequences of such dysregulation in not clear since behavioral performance on RMET in depressed patients was not significantly different from controls 
and did not appear biased towards negative affect. The task itself may not be difficult enough to tease out such biases (Domes et al., 2007). Whether these differences in functional activity are associated with state or trait markers also remains unclear.

Although we did not see any significant difference between groups with RMET contrast, we did find higher anterior subgenual cingulate activation and anterior temporal pole during RMET minus gender attribution in depressed, suggesting that mentalization preferentially linked processing of perceptual inputs to internal visceral emotional experiences (Figure 2). This contrasts with activations of anterior frontal pole, supramotor and working memory areas in healthy controls. Note that both groups exhibited activations of semantic and word processing regions but there too, activity was more restricted to the inferior frontal gyrus in depressed patients. These finding, along with a relatively faster reaction times in depressed patients, suggests that this cohort mentalized in a more primary, impulsive way, whereas in healthy controls the task engaged a more distributed network that involved higher order abstract cognitive and emotional integrative regions (Petrides and Pandya, 2007).

\section{THE EFFECTS OF OXYTOCIN ON MENTALIZATION}

Our fMRI data reveals that intra-nasal oxytocin has a differential effect upon the brain of depressed and normal people, as if in the state of depression, oxytocin "recruits" a more emotionally reflexive appraisal state that is less affectively reflective. Oxytocin increased primarily emotional circuits as in the cingulate (BA24) and insula in depressed patients during RMET. These increases appear to be in the same general directions as baseline activation patterns (Figure 3). Conversely, oxytocin increased ventral activity in healthy controls adding a different and substantial "primary affective appraisal" regional component (amygdala, parahippocampus) along with social appraisal (BA25 and 32) to baseline activation pattern (Figure 4). This is in contrast with reports of reduced activation of the amygdala in response to fear-inducing visual stimuli coupled with oxytocin inhalation (Kirsch et al., 2005). Oxytocin also moderately decreased the reaction time in healthy controls. This is in line with reports of oxytocin improving RMET in healthy individuals when analyses focused on the more complex and difficult items (Domes et al., 2007), In contrasts, depressed patients remained faster at their responses than controls but tended to show a more deliberative response to the situations. Oxytocin did not alter the accuracy of responses in either group.

\section{REFERENCES}

Anisman, H. (1984). "Vulnerability to depression: contribution of stress," in Neurobiology of Mood Disorders eds R. M. Post and J. C. Ballenger (Baltimore: Williams and Wilkins), 407-431.

Baron-Cohen, S., Ring, H., Chitnis, X., Wheelwright, S., Gregory, L., Williams, S., Brammer, M., and Bullmore, E. (2006). fMRI of parents of children with Asperger syndrome: a pilot study. Brain Cogn. 61, 122-130.

Baron-Cohen, S., Wheelwright, S., Hill, J., Raste, Y., and Plumb, I. (2001). The "Reading the Mind in the Eyes" test revised version: a study with normal adults, and adults with Asperger syndrome or high-functioning autism. J. Child. Psychol. Psychiatry 42, 241-251.

Bell, C. (2006). Plasma oxytocin levels in depression and their correlation with the temperament dimension of reward dependence. J. Psychopharmacol. 20, 656-660.

Burbach, J.P., Schoots, O., and Hernando, F. (1998). Biochemistry of vasopressin fragments. Prog. Brain Res. 119, 127-136.

Choi-Kain, L. W., and Gunderson, J. G. (2008). Mentalization: ontogeny, assess-

\section{LIMITATIONS}

Number of subjects is relatively small although the total scans analyzed is 80 . Individual variability and total sample size cannot identify main responders. The exclusion of one male subject in healthy controls did not significantly change the results. The effect size on behavioral response (RT) is small and perhaps due to relatively small single dose of oxytocin and the intra-nasal route. Added to this are the small size of the olfactory nasal mucosa, the small instillation volume and the relatively fast turnover of cerebrospinal fluid which will render the total amount of active oxytocin that reached the brain small (Pontiroli, 1998; Petrides and Pandya, 2007). Oxytocin is also known to gradually degrade into several metabolites that may reduce the potency of the preparation (Claybaugh and Uyehara, 1993; Burbach et al., 1998). The effect size on RT may have been influenced by the relative simple to moderate difficulty in the RMET images themselves which may limited the range of improvement after oxytocin inhalation (Domes et al., 2007).

In summary, we have shown that depressed subjects perform a mental attribution task differently than healthy matched controls. In addition, a single dose of intra-nasal oxytocin has distinct effects on functional activity of networks involved in a mental attribution task and enhances cognitive and emotional appraisal. These effects appear to be dependent on the distress levels of individuals, their likely capacity to empathize and the underlying homeostasis of the affective primary brain processes. It is not clear from our study what role, if any, oxytocin may have in modulating and perhaps treating depressive symptoms. Other studies of this important component of depressive pathology are needed with likely longer exposure and repeated oxytocin administration.

\section{ACKNOWLEDGMENTS}

Study funded by the Hope for Depression Research Foundation (ZN). Study was also made possible with general funds from NIMH 1K08MH70615-01A1 (ZN), the Mood Disorders Program, the Brain Stimulation Laboratory, the Center for Advanced Imaging Research at the Medical University of South Carolina (MUSC). The authors would like to thank Lee-Silsby Pharmacy in Cleveland, Ohio for providing the intra-nasal oxytocin and matching placebo, Kimberly Porter, Rph at MUSC Investigational Drug Services for randomization and dispensing drug, and the South Carolina Research Authority in Charleston, South Carolina for imaging storage and support.

ment, and application in the treatment of borderline personality disorder. Am J. Psychiatry 165, 1127-1135.

Claybaugh, J.R., and Uyehara, C. F. (1993) Metabolism of neurohypophysial hormones. Ann. N. Y. Acad. Sci. 689, 250-268.

de Leeuw, J., and Kreft, I. G. (1995). Questioning multilevel models. J. Educ. Behav. Stat. 20, 171-189.

Ditzen, B., Neumann, I. D., Bodenmann, G., von Dawans, B., Turner, R.A., Ehlert, U., and Heinrichs, M. (2007). Effects of different kinds of couple interaction on cortisol and heart rate responses to stress in women. Psychoneuroendocrinology 32, 565-574.

Domes, G., Heinrichs, M., Michel, A., Berger, C., and Herpertz, S. (2007). Oxytocin improves "mind-reading" in humans. Biol. Psychiatry 61, 731-733.

Epperson, C. N., McDougle, C. J., and Price, L. H. (1996a). Intranasal oxytocin in obsessive-compulsive disorder. Biol. Psychiatry 40, 547-549.

Epperson, C. N., McDougle, C. J., and Price, L. H. (1996b). Intranasal oxytocin in trichotillomania. Biol. Psychiatry 40, 559-560. 
Fonagy, P., and Target, M. (2007). The rooting of the mind in the body: new links between attachment theory and psychoanalytic thought. J. Am. Psychoanal. Assoc. 55, 411-456.

Gallagher, H. L., Happe, F., Brunswick, N., Fletcher, P.C., Frith, U., and Frith, C. D. (2000). Reading the mind in cartoons and stories: an fMRI study of 'theory of mind' in verbal and nonverbal tasks. Neuropsychologia 38, 11-21.

George, M. S., Kimbrell, T.A., Parekh, P. I., Ketter, T. A., Pazzaglia, P. J., Callahan, A., Frye, M., Marangell,L., Herscovitch, P., and Post, R. M. (1995). Actively Depressed Subjects Have Difficulty Inducing, and Blunted Limbic rCBF During, Transient Sadness. Miami:APA New Research Abstracts 99-NR167.

Green, M. J., Cahill, C. M., and Malhi, G. S. (2007). The cognitive and neurophysiological basis of emotion dysregulation in bipolar disorder. J. Affect. Disord. 103, 29-42.

Guastella, A. J., Mitchell, P. B., and Dadds, M. R. (2008). Oxytocin increases gaze to the eye region of human faces. Biol. Psychiatry 63, 3-5.

Happe, F., Ehlers, S., Fletcher, P., Frith, U., Johansson, M., Gillberg, C., Dolan, R., Frackowiak, R., and Frith, C. (1996). 'Theory of mind' in the brain. Evidence from a PET scan study of Asperger syndrome. Neuroreport 8, 197-201.

Happe, F. G. (1994). An advanced test of theory of mind: understanding of story characters' thoughts and feelings by able autistic, mentally handicapped, and normal children and adults. J. Autism Dev. Disord. 24, 129-154.

Hartmann, H. P. (2009). Psychoanalytic self psychology and its conceptual development in light of developmental psychology, attachment theory, and neuroscience. Ann. N. Y. Acad. Sci. 1159, 86-105.

Hoffman, D. A., and Gavin, M. B. (1998). Centering decisions in hierarchical linear models: implications for research in organizations. J. Manage. 24, 623-641.

Hurlemann, R., Patin, A., Onur, O. A., Cohen, M. X., Baumgartner, T., Metzler, S., Dziobek, I., Gallinat, J., Wagner, M., Maier, W., and Kendrick, K.M. (2010). Oxytocin enhances amygdala-dependent, socially reinforced learning and emotional empathy in humans. J. Neurosci. 30, 4999-5007.

Jenkinson, M., Bannister, P., Brady, M., and Smith, S. (2002). Improved optimization for the robust and accurate linear registration and motion correction of brain images. Neuroimage $17,825-841$.
Jenkinson, M., and Smith, S. (2001). A global optimisation method for robust affine registration of brain images. Med. Image Anal. 5, 143-156.

Johnston, P. J., Stojanov, W., Devir, H., and Schall, U. (2005). Functional MRI of facial emotion recognition deficits in schizophrenia and their electrophysiological correlates. Eur. J. Neurosci. 22, 1221-1232.

Kirsch, P., Esslinger, C., Chen, Q., Mier, D., Lis, S., Siddhanti, S., Gruppe, H., Mattay, V.S., Gallhofer, B., and MeyerLindenberg, A. (2005). Oxytocin modulates neural circuitry for social cognition and fear in humans. $J$. Neurosci. 25, 11489-11493.

Kuo, M., Mohler, B., Raudenbush, S. L., and Earls, F. J. (2000). Assessing exposure to violence using multiple informants: application of hierarchical linear model. J. Child. Psychol. Psychiatry 41, 1049-1056.

Marsh, A. A., Yu, H. H., Pine, D. S., and Blair, R. J. (2010). Oxytocin improves specific recognition of positive facial expressions. Psychopharmacology (Berl) 209, 225-232.

Mayberg, H. S., Liotti, M., Brannan, S. K., McGinnis, S., Mahurin, R. K., Jerabek, P. A., Silva, J. A., Tekell, J. L., Martin, C. C., Lancaster, J.L., and Fox, P.T. (1999). Reciprocal limbic-cortical function and negative mood: converging PET findings in depression and normal sadness. Am. J Psychiatry 156, 675-682.

Mayer, J. D., Salovey, P., Caruso, D. R., and Sitarenios, G. (2003). Measuring emotional intelligence with the MSCEIT V2.0. Emotion 3, 97-105.

McGuigan, W.M., Katzev, A. R., and Pratt, C.C. (2003). Multi-level determinants of retention in a home-visiting child abuse prevention program. Child Abuse Negl. 27, 363-380.

Meins, E., Fernyhough, C., Wainwright, R., Das Gupta, M., Fradley, E., and Tuckey, M. (2002). Maternal mindmindedness and attachment security as predictors of theory of mind understanding. Child Dev. 73, 1715-1726.

Nelson, E. E., and Panksepp, J. (1998). Brain substrates of infant-mother attachment: contributions of opioids, oxytocin, and norepinephrine. Neurosci. Biobehav. Rev. 22, 437-452.

Nezlek, J. B., and Zyzniewski, L. E. (1998) Using hierarchical linear modeling to analyze grouped data. Grouped Dyn 2,313-320.

Northoff, G. (2007). Psychopathology and pathophysiology of the self in depression-neuropsychiatric hypothesis. J. Affect. Disord. 104, 1-14.

Ozonoff, S., Pennington, B. F., and Rogers, S. J. (1991). Executive function deficits in high-functioning autistic individuals: relationship to theory of mind. J. Child Psychol. Psychiatry 32, 1081-1105.

Panksepp, J. (2005). Affective consciousness: core emotional feelings in animals and humans. Conscious. Cogn $14,30-80$.

Petrides, M., and Pandya, D. (2007) Efferent association pathways from the rostral prefrontal cortex in the macaque monkey. J. Neurosci. 27, 11573-11586.

Pilowsky, T., Yirmiya, N., Arbelle, S., and Mozes, T. (2000). Theory of mind abilities of children with schizophrenia children with autism, and normally developing children. Schizophr. Res. 42, 145-155.

Pontiroli,A.E. (1998). Peptide hormones: review of current and emerging uses by nasal delivery. Adv. Drug Deliv. Rev. 29, 81-87.

Price, J. L., and Drevets, W. C. (2009) Neurocircuitry of mood disorders. Neuropsychopharmacology 35 192-216.

Raudenbush, S.W., and Chan,W.S. (1993) Application of a hierarchical linear model to the study of adolescent deviance in an overlapping cohort design.J. Consult. Clin. Psychol. 61, 941-951.

Rizzolatti, G., and Fabbri-Destro, M. (2008). The mirror system and its role in social cognition. Curr. Opin. Neurobiol. 18, 179-184.

Sliwinski, M. J., and Hall, C. B. (1998). Constraints on general slowing: a meta-analysis using hierarchical linear models with random coefficients. Psychol. Aging 13, 164-175.

Smith, S. M. (2002). Fast robust automated brain extraction. Hum. Brain Mapp. 17, 143-155.

Stuss, D. T., Gallup, G. G. Jr., and Alexander M.P. (2001). The frontal lobes are necessary for 'theory of mind'. Brain 124, 279-286.

Uekermann, J., Channon, S.,Lehmkamper, C., Abdel-Hamid, M., Vollmoeller,W. and Daum, I. (2008). Executive function, mentalizing and humor in major depression. J. Int. Neuropsychol. Soc. $14,55-62$.

Uvnas-Moberg, K. (1998). Oxytocin may mediate the benefits of positive social interaction and emotions. Psychoneuroendocrinology 23 819-835.

Vogeley, K., Bussfeld, P., Newen, A. Herrmann, S., Happe, F., Falkai, P. Maier, W., Shah, N. J., Fink, G. R. and Zilles, K. (2001a). Mind reading. neural mechanisms of theory of mind and self-perspective. Neuroimage 14, 170-181.
Vogeley, K., Bussfeld, P., Newen, A., Herrmann, S., Happe, F., Falkai, P., Maier, W., Shah, N. J., Fink, G. R., and Zilles, K. (2001b). Mind reading: neural mechanisms of theory of mind and self-perspective. Neuroimage 14, 170-181.

Williams, J. G., Allison, C., Scott, F. J., Bolton, P. F., Baron-Cohen, S., Matthews, F.E., and Brayne, C. (2008). The Childhood Autism Spectrum Test (CAST): sex differences. J. Autism Dev. Disord. 38, 1731-1739.

Winslow, J. T., and Insel, T. R. (2004). Neuroendocrine basis of social recognition. Curr. Opin. Neurobiol. 14, 248-253.

Woolrich, M. W., Ripley, B. D., Brady, M., and Smith, S. M. (2001). Temporal autocorrelation in univariate linear modeling of FMRI data. Neuroimage $14,1370-1386$.

Worsley, K. J. (2001). “Statistical analysis of activation images," in Functional MRI: An Introduction to Methods, P. Jezzard, P. M. Matthews and S. M. Smith (Oxford: Oxford University Press), 251-270.

Zak, P. J., Kurzban, R., and Matzner, W. T. (2005). Oxytocin is associated with human trustworthiness. Horm. Behav. $48,522-527$.

Conflict of Interest Statement: The authors declare that the research was conducted in the absence of any commercial or financial relationships that could be construed as a potential conflict of interest.

Received: 18 July 2010; paper pending published: 13 August 2010; accepted: 23 August 2010; published online: 12 October 2010. Citation: Pincus D, Kose S, Arana A, Johnson K, Morgan PS, Borckardt J, Herbsman T, Hardaway F, George MS, Panksepp J and Nahas Z (2010) Inverse effects of oxytocin on attributing mental activity to others in depressed and healthy subjects: a double-blind placebo controlled fMRI study. Front. Psychiatry 1:134. doi: 10.3389/fpsyt.2010.00134

This article was submitted to Frontiers in Neuropsychiatric Imaging and Stimulation, a specialty of Frontiers in Psychiatry.

Copyright (C) 2010 Pincus, Kose, Arana, Johnson, Morgan, Borckardt, Herbsman, Hardaway, George, Panksepp and Nahas. This is an open-access article subject to an exclusive license agreement between the authors and the Frontiers Research Foundation, which permits unrestricted use, distribution, and reproduction in any medium, provided the original authors and source are credited. 